\title{
Women recovering from social rejection: The effect of the person and the situation on a hormonal mechanism of affiliation
}

\author{
Korrina A. Duffy ${ }^{\mathrm{a}, *}$, Lasana T. Harris ${ }^{\mathrm{b}}$, Tanya L. Chartrand ${ }^{\mathrm{a}, \mathrm{c}}$, Steven J. Stanton ${ }^{\mathrm{d}}$ \\ a Department of Psychology \& Neuroscience, Duke University, United States \\ ${ }^{\mathrm{b}}$ Department of Experimental Psychology, University College London, United Kingdom \\ c Fuqua School of Business, Duke University, United States \\ d Department of Management \&'Marketing, Oakland University, United States
}

\section{A R T I C L E I N F O}

\section{Article history:}

Received 30 July 2016

Received in revised form

14 November 2016

Accepted 15 November 2016

\section{Keywords:}

Rejection

Motivation

Affiliation

Withdrawal

Progesterone

Cortisol

\begin{abstract}
A B S T R A C T
Rejection can motivate either affiliation or withdrawal. In order to study how personality and situational variables influence whether women will be motivated to affiliate versus withdraw, we manipulate social feedback (rejection vs. acceptance) and opportunity for face-to-face interaction (blocked vs. face-toface) and measure the individual difference variables rejection sensitivity and social anxiety. We test how these variables affect endogenous progesterone and cortisol concentrations, which are presumed to signal motivational responses to rejection. We find that three-way interactions involving social feedback, opportunity for face-to-face interactions, and either social anxiety or rejection sensitivity significantly predict progesterone change, but not cortisol change. Both interactions are driven by sharp progesterone decreases for women high in social anxiety/rejection sensitivity who have been rejected and who have no opportunity to reaffiliate in a face-to-face interaction. This progesterone change may be a physiological marker of motivation for social avoidance following rejection for women who cannot reaffiliate and who are particularly socially anxious or sensitive to rejection.
\end{abstract}

Published by Elsevier Ltd.

\section{Introduction}

When we have been rejected, we may be motivated to affiliate with others in order to reconnect or to withdraw in order to avoid further rejection. Both motivations are reasonable following rejection - so what predicts whether we will affiliate versus withdraw, and how does physiology underlie our motivation and behavior? This study tests how personality and situational variables affect motivational responses to rejection, which we measure by changes in progesterone and cortisol, hormones tied to affiliation (Schultheiss et al., 2004, 2003) and stress (Dickerson and Kemeny, 2004), respectively.

Rejection is a very powerful negative experience. It leads to psychological and physiological distress, characterized by increases in negative affect and release of the stress hormone cortisol (Blackhart et al., 2007; Stroud et al., 2002). Because rejection is such a negative experience, it is a powerful social motivator which may motivate people to affiliate with (Maner et al., 2007) or to withdraw from oth-

\footnotetext{
* Corresponding author at: Duke University, Department of Psychology \& Neuroscience, 417 Chapel Drive, Durham, NC 27708-0086, United States.

E-mail address: korrina.duffy@duke.edu (K.A. Duffy).
}

ers (Goetz and Dweck, 1980; Molden and Maner, 2013; Richman and Leary, 2009). For example, after recalling a past experience involving rejection, participants indicated greater interest in making new friends than those who had recalled a past experience involving acceptance (Maner et al., 2007). In another study, participants receiving bogus feedback that they may end up alone later in life indicated a stronger desire to work with others in a subsequent task compared to those who received feedback predicting acceptance or an accident-prone future (Maner et al., 2007). In contrast, Goetz and Dweck (1980) showed that although some children chose to attempt reaffiliation following rejection, others socially withdrew - a choice largely driven by forecasting future rejection. Thus, people respond in two very different ways to the experience of rejection - affiliating on the one hand or withdrawing on the other.

Given these two opposing reactions to rejection, what determines how a given person responds? The Multimotive Model provides a framework for understanding these conflicting social motives emerging from rejection, and identifies moderators that predict which motive will be acted upon (Richman and Leary, 2009). One important moderator is the rejected person's expectations of social repair with the perpetrator of rejection. When expectations of social repair are low, rejected people are motivated to withdraw, 
but when expectations of social repair are high, rejected people are motivated to reaffiliate.

Although Richman and Leary (2009) focus on whether a rejected person affiliates with or withdraws from the perpetrator of rejection specifically, Martin (2015) extends this framework beyond the dyad, arguing that expectations of affiliation with others more generally moderates which motivation is activated for the rejected person. Along these lines, Maner et al. (2007) demonstrate that rejected participants only seek affiliation with new others who are realistic sources of affiliation, such as new face-to-face interaction partners. This finding makes sense given that research shows that people more effectively build affiliation in face-to-face interactions than in side-by-side or computer-mediated interactions (Drolet and Morris, 2000; Okdie et al., 2011). Thus, the social context affects whether rejected people are motivated to affiliate or withdraw.

Most rejection studies seeking a physiological underpinning of the rejection experience have focused on cortisol (Blackhart et al., 2007; Gunnar et al., 2003; Stroud et al., 2002), but another hormone, progesterone, may also play a critical role in reaffiliation following rejection. In multiple studies, progesterone has been linked to affiliation motivation (Brown et al., 2009; Schultheiss et al., 2003, 2004). In one study, progesterone was measured in women at three different time points across the menstrual cycle (Schultheiss et al., 2003). At each time point, participants also completed an implicit measure of affiliation motivation called the Picture Story Exercise. For this measure of affiliation motivation, participants write a story about what they think people are doing in photographs in which the context is ambiguous (i.e. three people talking on the street could be friends reconnecting or executives discussing a business deal). Affiliation motivation is measured as the frequency with which the participant uses affiliation-related imagery in his or her stories. In the study, women with higher average progesterone showed greater affiliation motivation (Schultheiss et al., 2003). Furthermore, as a woman's progesterone rose over the menstrual cycle, so too did her affiliation motivation. Another study experimentally increased affiliation motivation via a 30-min film clip and tested how this affected progesterone (Schultheiss et al., 2004). Importantly, when affiliation motivation was experimentally elevated, participants experienced an increase in progesterone. Taken together, these results provide evidence that affiliation motivation increases progesterone and that progesterone could potentially be thought of as a physiological index of affiliation motivation.

Given that increasing affiliation motivation leads to a release of progesterone, it follows that other ways of inducing affiliation motivation, such as rejection, may also change endogenous progesterone concentrations. Maner et al. (2010) found that social exclusion decreased progesterone in individuals with high social anxiety relative to a control condition. They found the opposite effect for individuals with high rejection sensitivity; that is, social rejection increased progesterone in individuals with high rejection sensitivity relative to a control condition. Maner et al. (2010) interpreted progesterone as a physiological marker of approachavoidance motivation following social exclusion/rejection, with those high in social anxiety tending toward avoidance and those high in rejection sensitivity tending toward approach.

Despite these intriguing initial findings, there remain some significant gaps in the literature that we attempt to address. First, studying the dynamics of both cortisol and progesterone following rejection within the same participants is important, but remains underexplored. Cortisol has been associated with psychosocial distress whereas progesterone has been associated with affiliation. Studying both hormones following rejection and a subsequent opportunity for face-to-face interaction - a situation that involves both stress and affiliation - allows us to dissociate their roles within the same social context. Unlike cortisol, progesterone should be sensitive to affiliation-related situational and personality variables that would affect affiliation motivation. Second, although research suggests that an opportunity for face-to-face interaction affects affiliation motivation following rejection, no research has tested its effect on progesterone. Linking face-to-face interaction and progesterone would provide evidence for a physiological mechanism through which face-to-face interactions increase affiliation and, more broadly, human connection. We also test the theory put forth in the Multimotive Model that expectancies of social repair affect motivational and behavioral reactions to rejection (Richman and Leary, 2009). In this study, we take this model beyond the dyad to test how interacting with a novel person affects progesterone and cortisol in recently rejected participants.

In line with Maner et al. (2007), we hypothesize that when there is an opportunity to reaffiliate face-to-face, this should promote affiliation motivation particularly for rejected participants, reflected in progesterone increases. When there is no opportunity to reaffiliate face-to-face, this should promote withdrawal motivation, reflected in progesterone decreases. Consistent with Maner et al. (2010), this should be the case particularly for rejected participants who are most negatively affected by rejection. To address these questions, the present study tests how social feedback (rejection vs. acceptance) and opportunity for face-to-face interaction (blocked vs. face-to-face) interact with individual difference variables rejection sensitivity and social anxiety to affect changes in progesterone, which we measure as a physiological marker of affiliation motivation.

\section{Materials and methods}

\subsection{Study design}

Our design allowed female participants to be either socially rejected or socially accepted by three female confederates (see Fig. 1). Participants then interacted with a new female confederate. The interaction with the new confederate occurred either face-to-face or behind a barrier, such that they could not see each other. This manipulated whether or not participants had an opportunity to reaffiliate in a face-to-face interaction. We collected saliva from participants at multiple time points throughout the experiment to measure progesterone and cortisol change (Schultheiss and Stanton, 2009). We also measured individual difference variables, including social anxiety and rejection sensitivity, which we hypothesized would affect motivation to affiliate or withdraw depending on the social context.

We decided to test our hypothesis specifically in women. Finding an effect in women represents a more conservative test of our hypothesis. This is because women have higher means and standard deviations of progesterone which make it is more difficult to find an effect (Wirth et al., 2007).

\subsection{Participants}

We recruited female participants not using hormonal contraceptives $\left(N=106 ; M_{\text {age }}=21.2\right.$ years $)$ through a university subject pool for a $90-$ min study. Participants were compensated $\$ 20$. Since hormones can be affected by various behaviors, participants were instructed not to eat food or drink caffeine or alcohol $2 \mathrm{~h}$ before the study, not to smoke $6 \mathrm{~h}$ before the study, not to exercise $12 \mathrm{~h}$ before the study, and not to have sexual intercourse $24 \mathrm{~h}$ before the study (Schultheiss and Stanton, 2009). All participants provided written informed consent and were debriefed at the end of the session. This study received approval from the appropriate local ethics committee and complied with national legislation and the Code of Ethical Principles for Medical Research Involving Human Subjects of the World Medical Association (Declaration of Helsinki). 


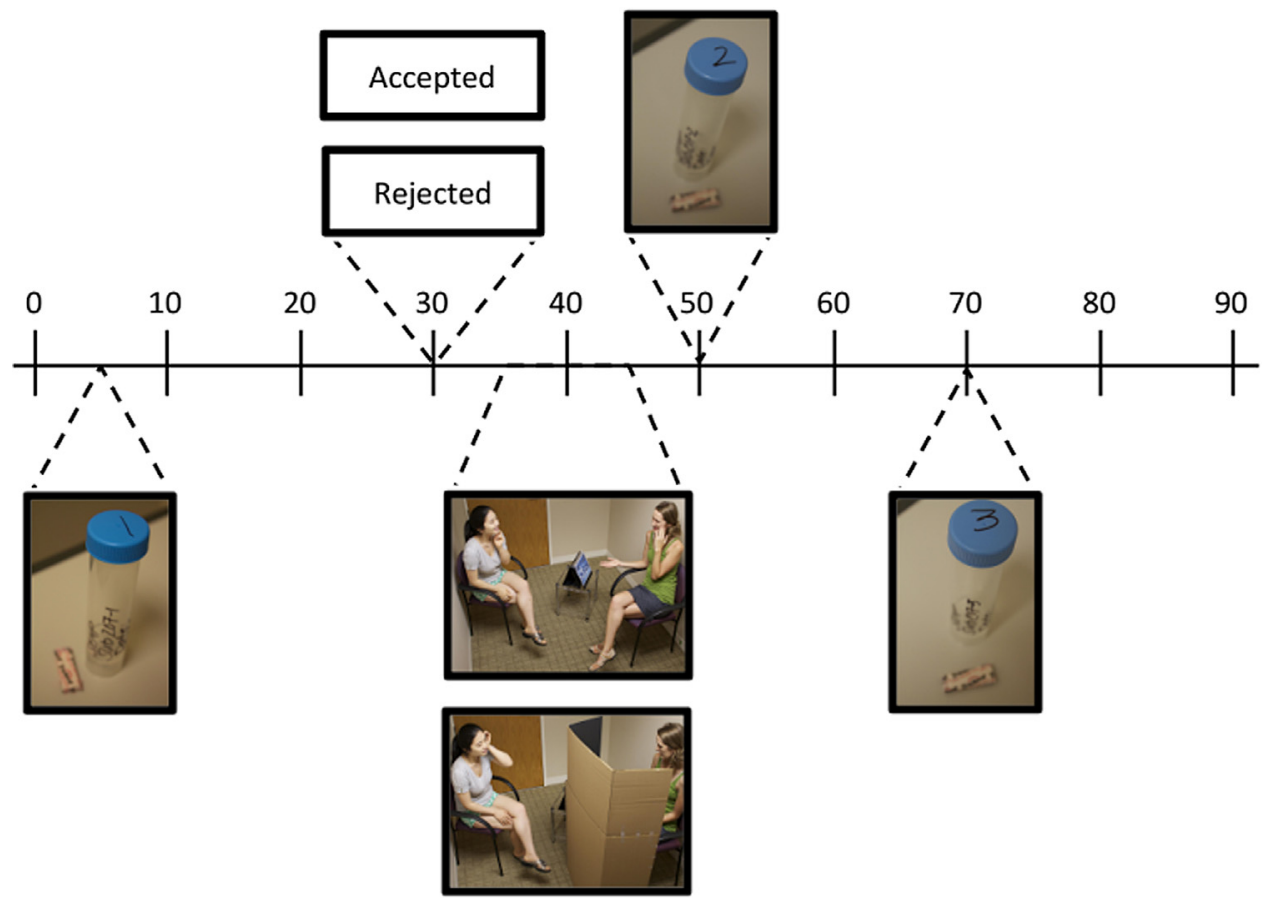

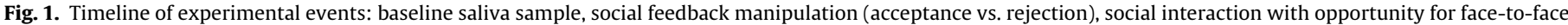

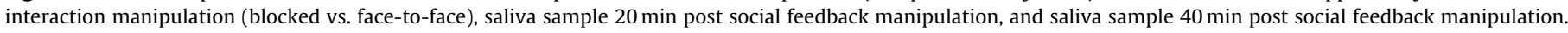

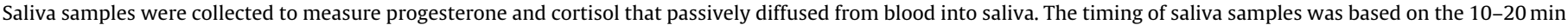
lag time that it takes for steroid hormones to be released into blood and then diffuse into saliva (Riad-Fahmy et al., 1987).

In order to estimate the sample size needed, we looked at the effect size found using a similar paradigm (Maner et al., 2010). In this study, the authors report the partial correlation of two critical interactions: the social feedback (control vs. exclusion) by social anxiety interaction on progesterone change, $B=-0.28$, $p=0.05, p r=-0.28$, and the social feedback (control vs. rejection) by rejection sensitivity interaction on progesterone change, $B=0.27$, $p=0.05, p r=0.28$. Based on a partial correlation of $|.28|$, we used $\mathrm{G}^{*}$ Power to calculate the sample size required in order to have a power of 0.80 with an alpha of 0.05 given this effect size. From this, we estimated a sample size of 95 . Since we anticipated that we might need to exclude outlier cases, we intended to collect data for 100 participants. For scheduling reasons, we ended up with 106 participants rather than the target of 100 participants. After excluding one participant from data analysis for having progesterone levels that were greater than 3 standard deviations above the mean, we had a final sample of 105 participants.

\subsection{Procedure}

\subsubsection{Cover story}

Participants signed up for a study entitled 'Impression Formation.' At the lab, the experimenter told participants that the study investigated how hormones affect impression formation. This was intended to explain both why we measure hormones (ask for saliva samples) and why we give participants social feedback (see next section). In order to encourage participants to believe that there were three other participants providing social feedback, participants were walked down a hall with four closed doors that led to study rooms. Participants were led into one room and told that other participants in the study were in the other three rooms.

\subsubsection{Manipulation of social feedback}

Participants were randomly assigned to one of two social feedback conditions (rejection vs. acceptance), which were manip- ulated using a paradigm adapted from previous work (Leary et al. 1995). In our paradigm, the experimenter told participants that the study was investigating impression formation, which required participants to give their impressions of one another. In order to do this, the experimenter instructed participants to talk about the things that they liked and disliked about themselves in a 1-2 min video designed to promote self-disclosure. After the participant finished recording the video on Windows Movie Maker, the experimenter returned to help upload the video onto Dropbox. The experimenter slowly uploaded the three confederate videos onto Dropbox to give the impression that the other participants were finishing the task at different times. When all four videos were uploaded to Dropbox, the experimenter gave the participant three feedback forms and instructed her to fill out the forms based on her impressions of the 'participants' in the three confederate videos. The experimenter assured the participant that the feedback would be anonymous. The feedback form consisted of five items: "I would introduce this person to a friend"; "I would continue talking to this person"; "I would invite this person to a party"; "This person reminds me of some of my own friends"; and "I would choose this person as a study partner in a difficult class". For each item, the participant selected a response (yes, unsure, no).

After the participant finished filling out the feedback forms, the experimenter came back into the room and collected the feedback forms in manila envelopes, one for each subject. The experimenter told the participant that she needed to collect the remaining forms and would return with the feedback as soon as possible. When the experimenter returned, the experimenter delivered prearranged feedback that manipulated either social rejection or social acceptance. The experimenter told the participant to look over her feedback and subsequently left the room. In the social rejection condition, the three feedback forms reflected a relatively negative impression of the participant, with answers of mostly 'unsure' and 'no' on the feedback forms (yes $=1$, unsure $=10$, no $=4$ ). In the social acceptance condition, the three feedback forms reflected a posi- 
tive impression of the participant, with answers of mostly 'yes' on the feedback forms (yes $=13$, unsure $=2$, no $=1$ ). As an important note, the confederates who had supposedly provided the manipulated feedback were all female, though representative of diverse racial groups. This ensured that female participants felt rejected or accepted by females generally rather than by a specific racial or ethnic group. After the participant looked over the feedback, the experimenter returned to inform the participant that she would complete the photo description task next.

\subsubsection{Manipulation of opportunity for face-to-face interaction}

In order to manipulate whether participants had an opportunity to reaffiliate with another person in a face-to-face interaction, participants were randomly assigned to either (1) the face-to-face condition in which they interacted face-to-face with a confederate or (2) the blocked condition in which face-to-face interaction was blocked by a cardboard barrier between the confederate and the participant. Including this manipulation allows us to test the effect of an opportunity to reaffiliate in a face-to-face interaction on progesterone change.

The social interaction was structured around a photo description task, which is a commonly used paradigm in studies involving social interactions (Chartrand and Bargh, 1999; Lakin and Chartrand, 2003; Lakin et al., 2008). In the task, the participant and the confederate take turns describing photos to one another for eight minutes total. Each photo was described for $1 \mathrm{~min}$ until a timer beeped, at which point the partners switched listening and describing roles. The room setup consisted of two chairs facing each other at a $90^{\circ}$ angle and situated between the chairs was a table holding a photo stand, which displayed photos to only the photo describer. Photo stimuli consisted of eight neutral nature scenes selected from the IAPS (Lang et al., 1997). One way to measure affiliation is by how much someone mimics another person (Chartrand and Bargh, 1999; Lakin and Chartrand, 2003; Lakin et al., 2008) so we measured mimicry as a behavioral measure of affiliation as well. Although we replicated the basic mimicry effects reported in Lakin and Chartrand (2003), we did not find new contributions to the literature so will not discuss this behavioral measure further.

\subsubsection{Hormone collection}

Testing was done between 12:00 pm and 5:00 pm to minimize diurnal variation in hormones. Each participant provided three saliva samples to be assayed for progesterone and cortisol. The participant provided the first sample upon arrival, the second sample 20 min post social feedback manipulation, and the third sample 40 min post social feedback manipulation. In order to appropriately time saliva sampling, participants engaged in a time estimation game as a filler task. We followed standard procedures developed for assaying hormones from saliva and samples were immediately sealed and placed in frozen storage post-collection (Dabbs, 1991; Gröschl, 2008; Schultheiss and Stanton, 2009).

\subsubsection{Questionnaire}

After providing the third saliva sample, participants completed a 15-min set of questionnaires on Qualtrics. First, participants completed the Hormone Assessment Questionnaire, which determined compliance to hormone collection instructions and addressed factors known to influence hormones (Schultheiss and Stanton, 2009). Then participants completed measures of individual differences: Social Phobia Scale (Mattick and Clarke, 1998), Rejection Sensitivity Scale (Downey and Feldman, 1996), Interpersonal Reactivity Index (Davis, 1980), and Need to Belong Scale (Leary et al., 2013). Next, participants reported demographic information. Then, as a manipulation check to assess whether the social feedback manipulation worked, participants were asked, "How did the feedback make you feel?" (5-point scale, $1=$ very bad, $5=$ very good). Finally, participants answered questions that assessed their suspicion regarding the feedback (Table 1 ).

\subsubsection{Hormonal assay procedure}

We used solid-phase Siemens Healthcare Diagnostics (Los Angeles, CA) Coat-A-Count ${ }^{125}$ I radioimmunoassays for cortisol (TKCO) and progesterone (TKPG). Following Liening et al., 2010; to determine salivary hormone concentrations, we prepared water-based dilutions of all standards and controls. Four hundred $\mu \mathrm{L}$ of the saliva samples, standards, and controls were pipetted into antibodycoated tubes. For progesterone, $1 \mathrm{~mL}$ radio-labeled tracer was subsequently added to each tube. All tubes were allowed to incubate overnight. For cortisol, $1 \mathrm{~mL}$ radio-labeled tracer was added to each tube following overnight incubation, and then all tubes were again incubated overnight. Finally, tubes were aspirated and counted for $3 \mathrm{~min}$. Analytical sensitivity, or the average lower limit of detection, was at $3.6 \mathrm{pg} / \mathrm{ml}$ (progesterone) and $.02 \mathrm{ng} / \mathrm{ml}$ (cortisol). Saliva samples were counted in duplicate and had a mean intra-assay coefficient of variation of $7.14 \%$ (progesterone) and $6.36 \%$ (cortisol).

\section{Results}

In order to correct for normality violations, we log-transformed raw progesterone and raw cortisol. For all progesterone analyses, we control for log-transformed progesterone at time 1 by including it as a predictor and we set log-transformed progesterone at time 3 as the outcome variable. For all cortisol analyses, we control for log-transformed cortisol at time 1 by including it as a predictor and we set log-transformed cortisol at time 3 as the outcome variable. ${ }^{1}$ Furthermore, for all hormone analyses, we control for the following variables: whether the participant had experienced bleeding gums, oral infections or oral lacerations over the past day as well as how many hours since the participant's last caffeine consumption. These variables, measured in the Hormone Assessment Questionnaire, were included as control variables in the analyses involving hormones. For all analyses, we report unstandardized betas.

Table 1

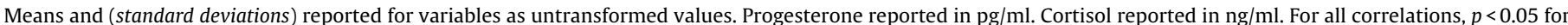
$r>|0.20|$ and $p<0.01$ for $r>0.25 \mid$.

\begin{tabular}{|c|c|c|c|c|c|c|c|c|c|c|c|}
\hline & & $\mathrm{M}(S D)$ & 1 & 2 & 3 & 4 & 5 & 6 & 7 & 8 & 9 \\
\hline 1 & Progesterone $\mathrm{T} 1$ & $56.1(43.8)$ & & & & & & & & & \\
\hline 2 & Progesterone T2 & $60.6(44.9)$ & 0.93 & & & & & & & & \\
\hline 3 & Progesterone $\mathrm{T} 3$ & $63.1(46.9)$ & 0.92 & 0.97 & & & & & & & \\
\hline 4 & Cortisol T1 & $2.0(1.3)$ & 0.11 & 0.11 & 0.09 & & & & & & \\
\hline 5 & Cortisol T2 & $1.9(1.3)$ & 0.02 & 0.17 & 0.14 & 0.51 & & & & & \\
\hline 6 & Cortisol T3 & $1.8(1.1)$ & -0.001 & 0.13 & 0.14 & 0.44 & 0.85 & & & & \\
\hline 7 & Rejection Sensitivity & $9.7(2.7)$ & -0.16 & -0.14 & -0.14 & 0.11 & 0.12 & 0.06 & & & \\
\hline 8 & Social Anxiety & $1.9(0.6)$ & 0.01 & 0.07 & 0.06 & -0.03 & 0.08 & 0.09 & 0.29 & & \\
\hline 9 & Need to Belong & $3.6(0.7)$ & -0.04 & -0.05 & -0.06 & 0.07 & -0.07 & -0.07 & 0.23 & 0.40 & \\
\hline 10 & Interpersonal Reactivity & $3.5(0.4)$ & 0.01 & -0.02 & -0.004 & 0.12 & -0.003 & -0.10 & 0.01 & 0.41 & 0.31 \\
\hline
\end{tabular}


Social Feedback on Hormone Change

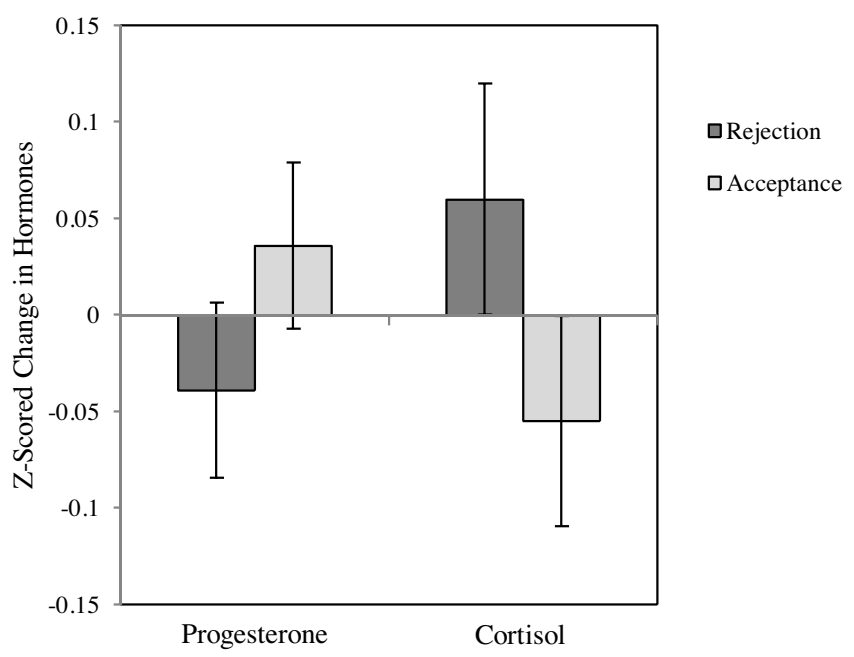

Fig. 2. The effect of social feedback (rejection vs. acceptance) on progesterone and cortisol change. Progesterone increased following acceptance relative to decreases following rejection. The opposite pattern was present for cortisol. Cortisol decreased following acceptance relative to increases following rejection. Error bars are 95\% confidence intervals. For the purposes of visualization, residual change scores for each hormone were calculated and then z-scored for this figure.

\subsection{Manipulation check}

In order to test the validity of the social feedback manipulation (rejection vs. acceptance), we ran an ANOVA with social feedback as our independent variable and self-reported reaction to feedback as our dependent variable. Rejected participants reported that the feedback made them feel relatively bad $(M=2.51, S D=0.62)$ whereas accepted participants reported that the feedback made them feel relatively good $(M=4.23, S D=0.76)$. Overall, rejected participants felt significantly worse than accepted participants, $F(1$, $97)=150.83 p<0.001, \eta^{2}=0.609$. For this analysis, we did not have data for all participants on the reaction to feedback variable because it was added after 7 participants had already completed the study.

We measured our individual difference variables, social anxiety and rejection sensitivity, at the end of the study after our manipulations. Because our measures of social anxiety and rejection sensitivity were intended to be trait variables, we ran ANOVAs to check statistically whether they were affected by our manipulations. Social feedback did not significantly affect social anxiety, $F(1,103)=0.03, p=0.87, \eta^{2}=0.0003$, or rejection sensitivity, $F(1$, $103)=0.97, p=0.33, \eta^{2}=0.009$. Opportunity for face-to-face interaction also did not significantly affect social anxiety, $F(1,103)=2.02$, $p=0.16, \eta^{2}=0.019$, or rejection sensitivity, $F(1,103)=1.66, p=0.20$, $\eta^{2}=0.016$. The interaction of social feedback and opportunity for face-to-face interaction also did not significantly affect social anxiety, $F(1,101)=0.02, p=0.89, \eta^{2}=0.0002$, or rejection sensitivity, $F(1,101)=1.15, p=0.29, \eta^{2}=0.011$. Furthermore, when we re-ran these analyses controlling for baseline cortisol and progesterone, social anxiety and rejection sensitivity were not significantly different between conditions.

\subsection{Effects of social feedback on hormone change}

Based on previous research suggesting that progesterone is a physiological marker of affiliation whereas cortisol is a physiological marker of stress, we predicted that when participants receive social feedback indicating acceptance by others, they should experience higher progesterone at time 3 relative to time 1 , and that when they receive social feedback indicating rejection by others, they should experience higher cortisol at time 3 relative to time 1 .

We ran a MANCOVA to test the effect of social feedback (rejection vs. acceptance) on two dependent variables, progesterone and cortisol at time 3 controlling for progesterone and cortisol at time 1. We used Wilk's lambda to assess multivariate effects. Using Wilk's lambda as the criterion, the composite dependent variate is significantly affected by social feedback, Wilk's lambda $=0.81$, $F(2,99)=11.75, p<0.001, \eta^{2}=0.74$. Univariate ANOVAs were conducted on progesterone change and cortisol change separately. There is a statistically significant effect of social feedback on both progesterone change, $F(1,98)=5.25, p=0.02, \eta^{2}=0.02$, and cortisol change, $F(1,98)=7.48, p=0.007, \eta^{2}=0.04$. The effect of social feedback on progesterone change is significant such that acceptance is associated with higher progesterone levels $(M=1.75 \mathrm{pg} / \mathrm{ml}$, $S E=0.02$ ) whereas rejection is associated with lower progesterone levels $(M=1.67 \mathrm{pg} / \mathrm{ml}, S E=0.02)$. The effect of social feedback on cortisol change is significant such that acceptance is associated with lower cortisol levels $(M=0.12 \mathrm{ng} / \mathrm{ml}, S E=0.03)$ whereas rejection is associated with higher cortisol levels $(M=0.23 \mathrm{ng} / \mathrm{ml}$, $S E=0.03$ ). (Means and standard deviations reported in log transformed progesterone and cortisol). In total, these results suggest that affiliation (in contrast to rejection) leads to higher progesterone levels whereas rejection (in contrast to affiliation) leads to higher cortisol levels (See Fig. 2).

\subsection{Opportunity for face-to-face interaction moderates effect of individual differences by social feedback on hormone change}

Based on hypotheses stemming from Maner et al. (2007) and Maner et al. (2010), we predicted that the interaction between individual differences and social feedback (rejection vs. acceptance) would be moderated by opportunity for face-to-face interaction (blocked vs. face-to-face) in predicting progesterone change. We tested these interactions separately for both individual difference variables, social anxiety and rejection sensitivity. In addition, we tested both models on cortisol change as well to assess whether the effects were specific to progesterone change. ${ }^{2}$

\subsubsection{Social anxiety}

We ran a multiple regression analysis with log-transformed progesterone at time $1, z$-scored social anxiety, social feedback (rejection $=0$, acceptance $=1$ ), opportunity for face-to-face interaction (blocked $=0$, face-to-face $=1$ ), and their interactions as the predictor variables and log-transformed progesterone at time 3 as the outcome variable. The resulting model is significant, $R^{2}=0.71, F(11,93)=20.39, p<0.001$. The three-way interaction between social anxiety, social feedback, and opportunity for faceto-face interaction is significant, $B=-0.17, t(93)=-2.66, p=0.009$, $S E=0.07$. The only marginally significant simple-simple effect of social anxiety on progesterone change is in the rejection/blocked condition, $B=-0.06, t(93)=-1.82, p=0.07, S E=0.03$, such that when participants are rejected and then interact behind a barrier, higher social anxiety predicts a decrease in progesterone. Two simple interactions are statistically significant. In the blocked condition, the simple interaction of social anxiety by social feedback is significant, $B=0.12, t(93)=2.31, p=0.02, S E=0.05$, such that higher social anxiety is associated with increases in progesterone in the acceptance condition but decreases in progesterone in the rejection condition. Critically, in the rejection condition, the simple interaction of social anxiety by opportunity for face-to-face interaction is significant, $B=0.11, t(93)=2.44, p=0.02, S E=0.04$, such that higher social anxiety is associated with increases in progesterone in the face-to-face condition but decreases in progesterone in the blocked condition. Thus, we see that in the rejection condition, the effect of 
Rejection Sensitivity x Social Feedback x Opportunity for Face-to-Face Interaction

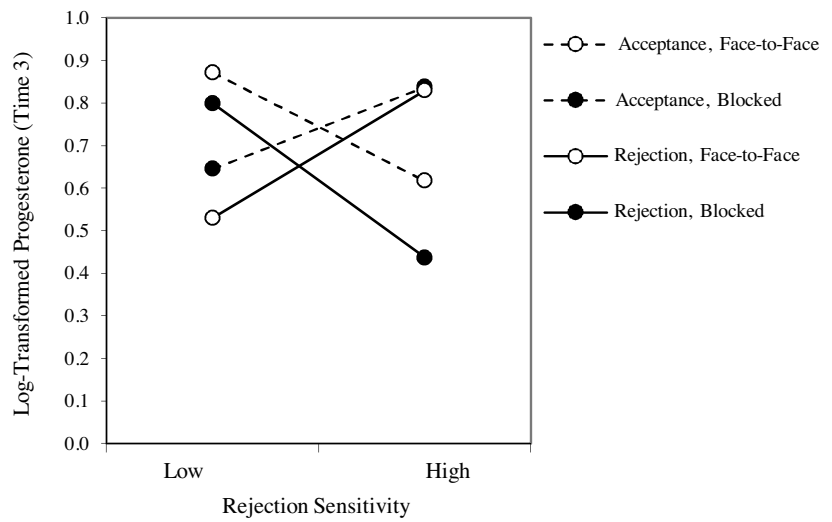

Social Anxiety x Social Feedback x Opportunity for Face-to-Face Interaction

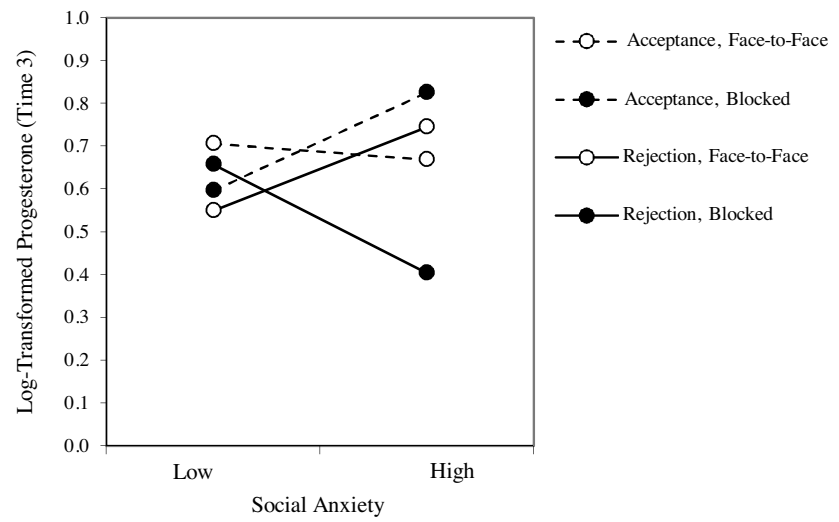

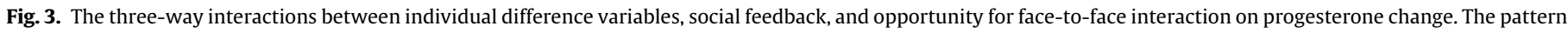

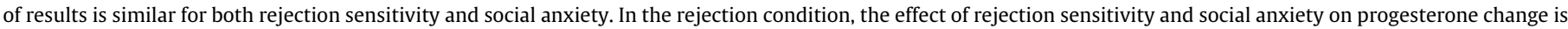

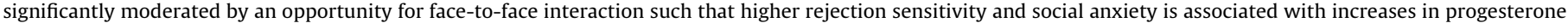
in the face-to-face condition but decreases in progesterone in the blocked condition.

social anxiety on progesterone change is significantly moderated by an opportunity for face-to-face interaction (See Fig. 3).

We then ran the same multiple regression analysis with cortisol change as the dependent variable. The resulting model is significant, $R^{2}=0.50, F(11,93)=8.50, p<0.001$, but the only significant effects are the covariates: log-transformed cortisol at time $1, B=0.64, t(93)=7.73, p<0.001, S E=0.08$, oral infections and lacerations, $B=-0.39, t(93)=2.63, p=0.01, S E=0.15$, and bleeding gums, $B=0.16, t(93)=2.38, p=0.02, S E=0.07$. The three-way interaction between social anxiety, social feedback, and opportunity for face-to-face interaction is not significant, $B=-0.11$, $t(93)=-1.36, p=0.18, S E=0.08$. Furthermore, all of the main effects and interaction effects involving social anxiety, social feedback, and opportunity for face-to-face interaction are also not significant.

\subsubsection{Rejection sensitivity}

We ran a multiple regression analysis with log-transformed progesterone at time $1, \mathrm{z}$-scored rejection sensitivity, social feedback (rejection $=0$, acceptance $=1$ ), opportunity for face-to-face interaction (blocked $=0$, face-to-face $=1$ ), and their interactions as the predictor variables and log-transformed progesterone at time 3 as the outcome variable. The resulting model is significant, $R^{2}=0.71, F(11,93)=20.81, p<0.001$. The three-way interaction between rejection sensitivity, social feedback, and opportunity for face-to-face interaction is significant, $B=-0.20, t(93)=-3.05$, $p=0.003, S E=0.06$. The only marginally significant simple-simple effect of rejection sensitivity on progesterone change is in the rejection/blocked condition, $B=-0.06, t(93)=-1.91, p=0.06, S E=0.03$, such that when participants are rejected and then interact behind a barrier, higher rejection sensitivity predicts a decrease in progesterone. Three simple interactions are statistically significant. In the blocked condition, the simple interaction of rejection sensitivity by social feedback is significant, $B=0.10, t(93)=2.08, p=0.04$, $S E=0.05$, such that higher rejection sensitivity is associated with increases in progesterone in the acceptance condition but decreases in progesterone in the rejection condition. Critically, in the rejection condition, the simple interaction of rejection sensitivity by opportunity for face-to-face interaction is significant, $B=0.12$, $t(93)=2.58, p=0.01, S E=0.05$, such that higher rejection sensitivity is associated with increases in progesterone in the face-to-face condition but decreases in progesterone in the blocked condition. Thus, we see again that in the rejection condition, the effect of rejection sensitivity on progesterone change is significantly moderated by an opportunity for face-to-face interaction. In order to test for simple interactions in the face-to-face condition, we recoded the opportunity for face-to-face interaction condition (face-to-face $=0$; blocked =1). In the face-to-face condition, the simple interaction of rejection sensitivity by social feedback is significant, $B=-0.10$, $t(93)=2.27, p=0.03, S E=0.04$, such that higher rejection sensitivity is associated with increases in progesterone in the rejection condition but decreases in progesterone in the acceptance condition (See Fig. 3). ${ }^{3}$

We then ran the same multiple regression analysis with logtransformed cortisol at time 3 as the dependent variable. The resulting model is significant, $R^{2}=0.47, F(11,93)=7.61, p<0.001$, but the only significant effects are the covariates: log-transformed cortisol at time $1, B=0.62, t(93)=7.27, p<0.001, S E=0.09$, oral infections and lacerations, $B=-0.39, t(93)=2.55, p=0.01, S E=0.15$, and bleeding gums, $B=0.14, t(93)=2.02, p=0.05, S E=0.07$. The threeway interaction between rejection sensitivity, social feedback, and opportunity for face-to-face interaction is not significant, $B=-0.05$, $t(93)=-0.59, p=0.56, S E=0.09$. Furthermore, all of the main effects and interaction effects involving rejection sensitivity, social feedback, and opportunity for face-to-face interaction are also not significant.

\section{Discussion}

The present research shows that social feedback affects endogenous progesterone and cortisol in different ways. Being rejected decreases progesterone and increases cortisol, whereas being accepted increases progesterone and decreases cortisol. These results support previous research showing that progesterone is associated with affiliation (Schultheiss et al., 2004, 2003; Wirth and Schultheiss, 2006), whereas cortisol is associated with psychosocial distress (Blackhart et al., 2007; Dickerson and Kemeny, 2004; Gunnar et al., 2009).

Importantly, the effect of social feedback is moderated by situational and personality variables. We find that the interaction between social feedback (rejection vs. acceptance), opportunity for face-to-face interaction (blocked vs. face-to-face), and individual difference variables (social anxiety, rejection sensitivity) significantly predicts progesterone change, but not cortisol change. We take this to mean that progesterone is uniquely affected by the affiliation manipulation (opportunity for face-to-face interaction) and individual difference variables that affect affiliation motivation (social anxiety and rejection sensitivity), allowing us to dissociate the roles of progesterone and cortisol in this social context.

When we further unpack the significant three-way interactions on progesterone change, we find that when women are 
rejected and interact face-to-face with a novel person, those higher in social anxiety/rejection sensitivity experience greater increases in progesterone, but when women are rejected and face-to-face interaction is blocked by a barrier, those higher in social anxiety/rejection sensitivity experience greater decreases in progesterone. Furthermore, we find that when face-to-face interaction is blocked, being socially accepted affects progesterone differently than being socially rejected. Accepted women higher in social anxiety/rejection sensitivity experience greater increases in progesterone whereas rejected women higher in social anxiety/rejection sensitivity experience greater decreases in progesterone, as mentioned above. Both of these interactions are driven by the fact that progesterone sharply decreases for those high in social anxiety/rejection sensitivity when they have been rejected and there is no opportunity to reaffiliate in a face-to-face interaction. This may signal a motivation for social avoidance, or withdrawal, following rejection for those who cannot reaffiliate face-to-face and who are particularly socially anxious and sensitive to rejection.

Maner et al. (2007) showed that rejected participants only seek reconnection with others who are a realistic source of social connection, defined as new interaction partners with whom actual face-to-face interaction is anticipated. Our results support their psychological findings with an additional layer of physiological evidence. Our study design allows us to test and demonstrate that face-to-face interactions affect the release of progesterone. Likely, face-to-face interactions affect progesterone because they facilitate affiliation and rapport (Chartrand and Bargh, 1999; Drolet and Morris, 2000; Lakin and Chartrand, 2003; Okdie et al., 2011). Faceto-face interaction allows for nonverbal behaviors that have been shown to increase rapport such as eye contact (Abramovitch and Daly, 1978), smiling (Tickle-Degnen et al., 1988), nodding (Muntigl et al., 2012), posture sharing (LaFrance and Broadbent, 1976), synchrony of movements (Hove and Risen, 2009; LaFrance, 1979; Ramseyer and Tschacher, 2011), and mimicry of gestures, mannerisms, and behaviors (Chartrand and Bargh, 1999; Lakin and Chartrand, 2003). Thus, when rejected people who are high in social anxiety or rejection sensitivity have an opportunity to engage in these nonverbal behaviors that help build affiliation, they have significantly greater increases in progesterone, a hormone associated with affiliation motivation, than when they do not have this opportunity. In this context, our results suggest that rejected people highest in social anxiety or rejection sensitivity who interact with a novel person face-to-face have high affiliation motivation, as indexed by increases in progesterone.

Interestingly, in contrast to Maner et al. (2010), we found that the effects of social anxiety and rejection sensitivity on progesterone change do not differ from one another. Our findings suggest that what is driving the difference in effects reported by Maner et al. (2010) is whether or not there is an opportunity for reaffiliation. In Study 1, Maner et al. (2010) interpret their finding that excluded participants high in social anxiety experience a decrease in progesterone to mean that those high in social anxiety tend to be motivated to withdraw following exclusion, but in that study, participants also did not have an opportunity for reaffiliation. If not having an opportunity for reaffiliation is actually what drove their effect, then their results are consistent with ours. In Study 2, Maner et al. (2010) interpret their finding that rejected participants high in rejection sensitivity experience an increase in progesterone to mean that those high in rejection sensitivity tend to be motivated to affiliate following rejection, but in this study, participants also had a perceived opportunity for reaffiliation in a face-to-face interaction. If having an opportunity for reaffiliation is what actually drove their effect, then, again, their results are in line with ours. Our results show that the effect of social anxiety and rejection sensitivity do not differ from one another, rather what seems to be driving the effects reported by Maner et al. (2010) is whether or not there was a realistic opportunity to reaffiliate following rejection.

Progesterone may serve a dual purpose of both facilitating affiliation and decreasing psychosocial distress (Wirth, 2011). We have made the case that progesterone is linked to affiliation, but we also want to emphasize its connection to stress reduction. Research has shown that progesterone decreases stress in animal studies (Bitran et al., 1995, 1993; Romeo et al., 1993). The mechanism by which progesterone decreases stress is by conversion of progesterone into allopregnanolone, a powerful anxiolytic that reduces stress in two ways: (1) by acting on GABA receptors in the brain and (2) by downregulating gene transcription for corticotrophin-releasing hormone in the HPA axis (Bitran et al., 1995, 1993; Patchev et al., 1996, 1994). Based on evidence from animal models, we can extrapolate from this research to suggest that in humans increases in progesterone likewise have the potential to reduce stress (Bitran et al., 1995, 1993; Patchev et al., 1994). The role of progesterone in decreasing psychosocial distress is particularly important to consider in the context of rejection and may facilitate approachoriented, affiliation motivation (Wirth, 2011). In fact, we speculate that the inverse relationship that we observed between cortisol and progesterone may have been caused by a downstream effect of progesterone on cortisol.

We would like to emphasize that this is the first study to examine the downstream effect of social rejection on progesterone and cortisol as participants engage in a social interaction with another person. This design allowed us to test how cortisol and progesterone respond in a motivationally tuned manner to the social context and individual differences that affect social motivations to either affiliate or withdraw. Thus, our study uniquely addresses the complexity of how the social context and individual differences affect hormone responses to social interactions following rejection. A previous study examined the effect of social exclusion on cortisol, progesterone, and testosterone, but used a computerized ball tossing game to manipulate exclusion and inclusion (Seidel et al., 2013). The manipulation did not affect cortisol, which might suggest that participants did not think that the manipulation was realistic. Furthermore, in women, but not men, both inclusion and exclusion led to increases in progesterone, which the authors speculate may reflect a general affiliative response during social interactions. By not using paradigms with actual face-to-face interactions with other people, it is difficult to understand how social exclusion and social rejection affect our physiology in the real world in which social contexts and individual differences matter.

Despite the contributions we have discussed, this study has a few limitations that should be kept in mind when interpreting the results. First, since we tested only women in our study, we cannot generalize our findings to men. While some research has shown that affiliation motivation increases progesterone in both men and women (Schultheiss et al., 2004), other research has demonstrated sex differences in the link between baseline progesterone and affiliative motivation (Schultheiss et al., 2003). Therefore, more research is needed to test how individual difference variables (social anxiety and rejection sensitivity) and opportunity for face-to-face interaction affect progesterone following rejection or acceptance in men. Second, we measured individual difference variables, social anxiety and rejection sensitivity, at the end of the study after our manipulations, which means they could have potentially been affected by our manipulations. Although we statistically confirmed that self-reported social anxiety and rejection sensitivity did not differ by manipulated condition, it would be preferable in future work to measure these trait variables before participants come to the lab or at least in advance of the manipulation. Third, based on the literature supporting the link between progesterone and affiliation motivation (Schultheiss et al., 2003, 2004), we measured progesterone as a marker of affiliation motivation rather than 
measuring affiliation motivation directly. Future research should measure self-reported or implicit affiliation motivation alongside progesterone to ensure that progesterone is indeed indexing affiliation motivation. Fourth, the average level of progesterone that we report is considered high and may be due the use of sugarless chewing gum to stimulate saliva flow (Schultheiss, 2013). Recent research has shown that sugarless chewing gum leads to higher reported levels of progesterone when measured using radioimmunoassays (Schultheiss, 2013). Although we used Trident original flavor sugarless chewing gum and Schultheiss (2013) used Orbit spearmint flavor sugarless chewing gum, the level of progesterone may have been artificially inflated by the use of sugarless chewing gum. Finally, social anxiety and rejection sensitivity are moderately correlated so future research should also measure constructs, such as negative affect and neuroticism, that may underlie both social anxiety and rejection sensitivity. Doing so would disambiguate whether negative affect or neuroticism can explain these presently-reported effects.

\section{Conclusions}

Overall, our results demonstrate the effect of social feedback, opportunity for face-to-face interaction, and individual difference variables on progesterone. We support previous findings that progesterone may serve as a physiological marker of approachavoidance motivation (Maner et al., 2010). When women have an opportunity to reaffiliate face-to-face, rejection increases progesterone particularly for those high in social anxiety and rejection sensitivity. Increases in progesterone may signal affiliation motivation and/or approach motivation. When women do not have an opportunity to reaffiliate face-to-face, rejection may decrease progesterone particularly in those high in social anxiety and rejection sensitivity. Decreases in progesterone may signal withdrawal and/or avoidance motivation. Our results emphasize how context plays a role in motivations for affiliation and withdrawal following rejection: for women high in social anxiety or rejection sensitivity, having an opportunity for reaffiliation in a face-to-face is critically important.

\section{Footnotes}

1. We have reported the results at time 3 rather than time 2 because the effects at time 2 were directionally consistent with time 3 only slightly weaker. This strengthening of the effect over time may be partially explained by the time delay steroid hormones experience passively diffusing into saliva after being released into the bloodstream.

2. For the individual difference measures, need to belong and interpersonal reactivity, we ran the same analyses that we included in our manuscript for social anxiety and rejection sensitivity. For log-transformed progesterone at time 3, the three-way interaction between need to belong, social feedback, and opportunity for face-to-face interaction is non-significant, $B=-0.09$, $t(93)=-1.38, p=0.17, S E=0.07$, and the three-way interaction between interpersonal reactivity, social feedback, and opportunity for face-to-face interaction is also non-significant, $B=-0.04$, $t(93)=-0.54, p=0.59, S E=0.07$. Likewise, for cortisol change, the three-way interactions are non-significant for need to belong, $B=-0.01, t(93)=-0.11, p=0.91$, and interpersonal reactivity, $B=-0.07, t(93)=-0.85, p=0.40, S E=0.09$.

3. When we run the analyses excluding participants who report taking any medication, it does not change the significance or pattern of results. Because progesterone varies across the menstrual cycle, we also statistically confirmed that menstrual cycle phase did not affect our results. In order to use menstrual cycle phase as a covariate, we created a categorical coding scheme for the early follicular phase (day 1 to day 7), late follicular phase (day 8 to day 14), and late luteal phase (day 15 and beyond). We also created a category for women who did not have a menstrual cycle. When we run the analyses controlling for menstrual cycle phase with this categorical covariate, it does not change the significance or pattern of results.

\section{Authorship}

K.A. Duffy, S.J. Stanton, T.L. Chartrand, and L.T. Harris developed the study concept and study design. K.A. Duffy performed data collection. K.A. Duffy performed the hormone analysis under the supervision of S.J. Stanton and K.A. Duffy performed the data analysis under the supervision of S.J. Stanton, T.L. Chartrand, and L.T. Harris. K.A. Duffy drafted the manuscript and T.L. Chartrand, S.J. Stanton, and L.T. Harris provided critical revisions. All authors approved the final version of the manuscript for submission.

\section{Conflicts of interest}

The authors have no conflicts of interest to declare.

\section{Acknowledgments}

The authors would like to thank Mark Leary for his help on the social rejection paradigm. Thank you to undergraduate research assistants Kaitlyn Batt, Chay'daa Mohammed, Maryann Verghese, and Olivia Chen for help running the study. Thank you to video confederates Simone Tang, Bree Gray-Jordan, and Libby Malcolm. Also, special thanks to Steven Shepherd and Peggy Liu for help with statistics.

\section{References}

Abramovitch, R., Daly, E.M., 1978. Children's use of head orientation and eye contact in making attributions of affiliation. Child Dev. 49, 519-522.

Bitran, D., Purdy, R.H., Kellogg, C.K., 1993. Anxiolytic effect of progesterone is associated with increases in cortical allopregnanolone and GABA-A receptor function. Pharmacol. Biochem. Behav, 45, 423-428.

Bitran, D., Shiekh, M., McLeod, M., 1995. Anxiolytic effect of progesterone is mediated by the neurosteroid allopregnanolone at brain GABA-A receptors. J. Neuroendocrinol. 7, 171-177.

Blackhart, G.C., Eckel, L.A., Tice, D.M., 2007. Salivary cortisol in response to acute social rejection and acceptance by peers. Biol. Psychol. 75, 267-276, http://dx. doi.org/10.1016/j.biopsycho.2007.03.005.

Brown, S.L., Fredrickson, B.L., Wirth, M.M., Poulin, M.J., Meier, E.a., Heaphy, E.D., Cohen, M.D., Schultheiss, O.C., 2009. Social closeness increases salivary progesterone in humans. Horm. Behav. 56, 108-111, http://dx.doi.org/10. 1016/j.yhbeh.2009.03.022.

Chartrand, T.L., Bargh, J.A., 1999. The chameleon effect: the perception-behavior link and social interaction. J. Pers. Soc. Psychol. 76, 893-910.

Dabbs, J.M., 1991. Salivary testosterone measurements Collecting, storing, and mailing saliva samples. Physiol. Behav. 49, 815-817.

Davis, M.H., 1980. A multidimensional approach to individual differences in empathy. JSAS Cat. Sel. Doc. Psychol. 10.

Dickerson, S.S., Kemeny, M.E., 2004. Acute stressors and cortisol responses: a theoretical integration and synthesis of laboratory research. Psychol. Bull. 130, 355-391, http://dx.doi.org/10.1037/0033-2909.130.3.355.

Downey, G., Feldman, S.I., 1996. Implications of rejection sensitivity for intimate relationships. J. Pers. Soc. Psychol. 70, 1327-1343.

Drolet, A.L., Morris, M.W., 2000. Rapport in conflict resolution: accounting for how face-to-face contact fosters mutual cooperation in mixed-motive conflicts. J. Exp. Soc. Psychol. 36, 26-50, http://dx.doi.org/10.1006/jesp.1999.1395.

Goetz, T., Dweck, C.S., 1980. Learned helplessness in social situations. J. Pers. Soc. Psychol. 39, 246-255.

Gröschl, M., 2008. Current status of salivary hormone analysis. Clin. Chem. 54, 1759-1769, http://dx.doi.org/10.1373/clinchem.2008.108910.

Gunnar, M.R., Sebanc, A.M., Tout, K., Donzella, B., van Dulmen, M.M.H., 2003. Peer rejection, temperament, and cortisol activity in preschoolers. Dev. Psychobiol. 43, 346-368.

Gunnar, M.R., Talge, N.M., Herrera, A., 2009. Stressor paradigms in developmental studies: what does and does not work to produce mean increases in salivary cortisol. Psychoneuroendocrinology 34, 953-967, http://dx.doi.org/10.1016/j. psyneuen.2009.02.010. 
Hove, M.J., Risen, J.L., 2009. It's all in the timing: interpersonal synchrony increases affiliation. Soc. Cogn. 27, 949-960, http://dx.doi.org/10.1521/soco.2009.27.6. 949.

LaFrance, M., Broadbent, M., 1976. Group rapport: posture sharing as a nonverbal indicator. Gr. Organ. Stud. 1, 328-333.

LaFrance, M., 1979. Nonverbal synchrony and rapport: analysis by the cross-lag panel technique. Soc. Psychol. 0. 42, 66-70.

Lakin, J.L., Chartrand, T.L., 2003. Using nonconscious behavioral mimicry to create affiliation and rapport. Psychol. Sci. 14, 334-339, http://dx.doi.org/10.1111/ 1467-9280.14481.

Lakin, J.L., Chartrand, T.L., Arkin, R.M., 2008. I am too just like you: nonconscious mimicry as an automatic behavioral response to social exclusion. Psychol. Sci. 19, 816-822.

Lang, P.J., Bradley, M.M., Cuthbert, B.N.,1997. International affective picture system (IAPS): Technical manual and affective ratings.

Leary, M.R., Tambor, E.S., Terdal, S.K., Downs, D.L., 1995. Self-esteem as an interpersonal monitor: the sociometer hypothesis. J. Pers. Soc. Psychol. 68, 518-530, http://dx.doi.org/10.1037//0022-3514.68.3.518.

Leary, M.R., Kelly, K.M., Cottrell, C.a., Schreindorfer, L.S., 2013. Construct validity of the need to belong scale: mapping the nomological network. J. Pers. Assess. 95, 610-624, http://dx.doi.org/10.1080/00223891.2013.819511.

Liening, S.H., Stanton, S.J., Saini, E.K., Schultheiss, O.C., 2010. Salivary testosterone cortisol, and progesterone: two-week stability, interhormone correlations, and effects of time of day, menstrual cycle, and oral contraceptive use on steroid hormone levels. Physiol. Behav. 99, 8-16, http://dx.doi.org/10.1016/j.physbeh. 2009.10.001.

Maner, J.K., DeWall, C.N., Baumeister, R.F., Schaller, M., 2007. Does social exclusion motivate interpersonal reconnection? Resolving the porcupine problem. J. Pers. Soc. Psychol. 92, 42-55, http://dx.doi.org/10.1037/0022-3514.92.1.42.

Maner, J.K., Miller, S.L., Schmidt, N.B., Eckel, L.A., 2010. The endocrinology of exclusion: rejection elicits motivationally tuned changes in progesterone. Psychol. Sci. 21, 581-588, http://dx.doi.org/10.1177/0956797610362676.

Martin, J., 2015. Social Expectancies and Multiple Goals: Explaining the Seemingly Incompatible Behavioral Responses to Social Rejection. Duke University, Durham, NC (Unpublished).

Mattick, R.P., Clarke, J.C., 1998. Development and validation of measures of social phobia scrutiny fear and social interaction anxiety. Behav. Res. Ther. 36, 455-470.

Molden, D.C., Maner, J.K., 2013. How and when exclusion motivates social reconnection. In: DeWall, N. (Ed.), Handbook of Social Exclusion. Oxford University Press.

Muntigl, P., Knight, N., Watkins, A., 2012. Working to keep aligned in psychotherapy: using nods as a dialogic resource to display affiliation. Lang. Dialogue 2, 9-27.

Okdie, B.M., Guadagno, R.E., Bernieri, F.J., Geers, A.L., Mclarney-Vesotski, A.R., 2011. Getting to know you: face-to-face versus online interactions. Comput. Human Behav. 27, 153-159, http://dx.doi.org/10.1016/j.chb.2010.07.017.

Patchev, V.K., Shoaib, M., Holsboer, F., Almeida, O.F., 1994. The neurosteroid tetrahydroprogesterone counteracts corticotropin-releasing hormone-induced anxiety and alters the release and gene expression of corticotropin-releasing hormone in the rat hypothalamus. Neuroscience $62,265-271$.
Patchev, V.K., Hassan, A.H., Holsboer, D.F., Almeida, O.F., 1996. The neurosteroid tetrahydroprogesterone attenuates the endocrine response to stress and exerts glucocorticoid-like effects on vasopressin gene transcription in the rat hypothalamus. Neuropsychopharmacology 15, 533-540.

Ramseyer, F., Tschacher, W., 2011. Nonverbal synchrony in psychotherapy: coordinated body-movement reflects relationship quality and outcome. J. Consult. Clin. Psychol. 79, 285-295, http://dx.doi.org/10.1037/a0023419.

Riad-Fahmy, D., Read, G.F., Walker, R.F., Walker, S.M., Grifiths, K., 1987. Determination of ovarian steroid hormone levels in saliva: an overview. J. Reprod. Med. 32, 254-272.

Richman, L.S., Leary, M.R., 2009. Reactions to discrimination, stigmatization, ostracism, and other forms of interpersonal rejection: a multimotive model. Psychol. Rev. 116, 365-383, http://dx.doi.org/10.1037/a0015250 (Reactions).

Romeo, E., Cavallaro, S., Korneyev, A., Kozikowski, A.P., Ma, D., Polo, A., Costa, E., Guidotti, A., 1993. Stimulation of brain steroidogenesis by 2-aryl-indole-3-acetamide derivatives acting at the mitochondrial diazepam-binding inhibitor receptor complex. J. Pharmacol. Exp. Ther. 267, 462-471.

Schultheiss, O.C., Stanton, S.J., 2009. Assessment of salivary hormones. In: Harmon-Jones, E., Beer, J.S. (Eds.), Methods in Social Neuroscience. The Guilford Press, New York, pp. 17-44.

Schultheiss, O.C., Dargel, A., Rohde, W., 2003. Implicit motives and gonadal steroid hormones: effects of menstrual cycle phase, oral contraceptive use, and relationship status. Horm. Behav. 43, 293-301, http://dx.doi.org/10.1016/ S0018-506X(03)00003-5.

Schultheiss, O.C., Wirth, M.M., Stanton, S.J., 2004. Effects of affiliation and power motivation arousal on salivary progesterone and testosterone. Horm. Behav. 46, 592-599, http://dx.doi.org/10.1016/j.yhbeh.2004.07.005.

Schultheiss, O.C., 2013. Effects of sugarless chewing gum as a stimulant on progesterone, cortisol, and testosterone concentrations assessed in saliva. Int. J Psychophysiol. 87, 111-114, http://dx.doi.org/10.1016/j.ijpsycho.2012.11.012.

Seidel, E.M., Silani, G., Metzler, H., Thaler, H., Lamm, C., Gur, R.C., Kryspin-Exner, I. Habel, U., Derntl, B., 2013. The impact of social exclusion vs: inclusion on subjective and hormonal reactions in females and males. Psychoneuroendocrin 38, 2925-2932.

Stroud, L.R., Salovey, P., Epel, E.S., 2002. Sex differences in stress responses: social rejection versus achievement stress. Biol. Psychiatry 52, 318-327.

Tickle-Degnen, L., Rosenthal, R., Harrigan, J.A., 1988. Nonverbal behavior as a determinant of favorableness of impressions formed: eight meta-analyses. Unpubl. Manuscr. Harvard Un.

Wirth, M.M., Schultheiss, O.C., 2006. Effects of affiliation arousal (hope of closeness) and affiliation stress (fear of rejection) on progesterone and cortisol. Horm. Behav. 50, 786-795, http://dx.doi.org/10.1016/j.yhbeh.2006.08.003.

Wirth, M.M., Meier, E.A., Fredrickson, B.L., Schultheiss, O.C., 2007. Relationship between salivary cortisol and progesterone levels in humans. Biol. Psychol. 74, 104-107, http://dx.doi.org/10.1016/j.biopsycho.2006.06.007.

Wirth, M.M., 2011. Beyond the HPA axis: progesterone-derived neuroactive steroids in human stress and emotion. Front. Endocrinol. 2, 1-14, http://dx.doi. org/10.3389/fendo.2011.00019. 